\title{
PI SZ phenotype in chronic obstructive pulmonary disease
}

\author{
L Alvarez-Granda, M J Cabero-Perez, A Bustamante-Ruiz, D Gonzalez-Lamuño, \\ M Delgado-Rodriguez, M Garcia-Fuentes
}

\begin{abstract}
Background - A study was undertaken to clarify whether the Pi SZ phenotype of the protease inhibitor system predisposes to chronic obstructive pulmonary disease (COPD).

Methods - The prevalence of PI Z and PI $S Z$ deficient phenotypes was investigated in a population of 702 patients with COPD followed up at the Chest Unit of a tertiary hospital and in 15400 newborn infants from the same geographical area. Individuals with deficiency were detected by screening of dried blood spots on filter paper using a comparative electroimmunodiffusion technique for $\alpha_{1}$-antitrypsin and transferrin. The serum phenotype was confirmed by means of isoelectrofocusing on polyacrylamide gel.

Results - Of the 702 blood samples from patients with COPD, six PI Z subjects $(0.85 \%)$ and one PI SZ $(0.14 \%)$ were detected. Of the 15400 samples from neonates, the number of $P I Z$ subjects was eight $(0.052 \%)$ and that of PI SZ was 24 $(0.156 \%)$. The difference between the two groups was significant for PI Z but not for PI SZ.
\end{abstract}

Conclusions - The data do not indicate an increased risk for development of COPD associated with the PI SZ phenotype but confirm the predisposition of $P I Z$ individuals for the development of COPD. (Thorax 1997;52:659-661)

Keywords: $\alpha_{1}$-antitrypsin, chronic obstructive pulmonary disease, neonates, screening.

Alpha $_{1}$-antitrypsin and its variants make up what is known as the protease inhibitor system (PI). Its deficiency causes pulmonary emphysema. ${ }^{1}$ Over 75 variants of the PI system have been recognised, some of which may produce a clinically significant reduction in levels of $\alpha_{1}$-antitrypsin. These variants are fundamentally the $\mathrm{Z}$ and $\mathrm{S}$ forms. Classically, deficient phenotypes are considered to be those which have plasma levels of $\alpha_{1}$-antitrypsin 35\% lower than the levels found in normal individuals. ${ }^{2}$ These phenotypes are PI SZ and PI $Z$. The association between PI Z and pulmonary emphysema is clearly established, as is the role of smoking as an aggravating factor of this disease in deficient individuals. ${ }^{3}$ Data on the PI SZ phenotype are inconclusive, with some studies ${ }^{45}$ concluding that the PI SZ phenotype involves a serious risk of development of pulmonary emphysema while others $^{67}$ fail to support this conclusion. The prevalence of this phenotype in a population of patients with COPD compared with that found in newborn infants from the same geographical area was investigated with the aim of clarifying whether the PI SZ phenotype predisposes to the development of chronic respiratory disease.

\section{Methods \\ STUDY POPULATION}

A patient was considered as eligible if he or she had a diagnosis of COPD (chronic bronchitis and emphysema) and was receiving medical care at the Chest Unit of Valdecilla Hospital in Cantabria (Spain) in 1993. Inclusion criteria were diagnosis of symptomatic COPD, forced expiratory volume in one second $\left(\mathrm{FEV}_{1}\right)$ less than $80 \%$ predicted, and a ratio of $\mathrm{FEV}_{1}$ to forced vital capacity (FVC) of less than $70 \%$. All the 702 patients eligible gave their consent to participate.

Between 1987 and 198915400 neonates in our region were also studied to determine the prevalence of $\alpha_{1}$-antitrypsin deficiency.

SCREENING METHOD

The technique used for screening was a modification of that described by Laurell $^{8}$ and consisted of a simultaneous electroimmunodiffusion assay for $\alpha_{1}$-antitrypsin and transferrin in the following stages: (1) elution of $40 \mathrm{~mm}^{2}$ of filter paper impregnated with capillary blood in $75 \mu \mathrm{l}$ saline serum $(0.9 \%)$ for 24 hours; (2) the eluate ( $5 \mu \mathrm{l}$ per sample) is subjected to electrophoresis on agar gel containing anti- $\alpha_{1-}$ antitrypsin $(5.8 \%)$ and anti-transferrin $(5 \%)$ antibodies (Behring), with the application of a continuous voltage of 10 volts $/ \mathrm{cm}$ for four hours. The plates were then stained with Coomassie Blue.

The result of the electrophoresis corresponded to two peaks delimited by the antigen-antibody precipitation lines. The concentration of antisera in the gel was such that, in samples from normal non- $\alpha_{1}$-antitrypsin deficient individuals, the two peaks attain similar heights. The samples from patients with severe $\alpha_{1}$-antitrypsin deficiency produced a transferrin peak of normal height and a very low $\alpha_{1}$-antitrypsin peak. To avoid failure to detect PI SZ individuals, samples suspected of being deficient were considered to be those in which the ratio of the heights of the $\alpha_{1}$-antitrypsin/ transferrin peaks was less than or equal to 0.65 . This cut-off point was chosen after the finding 
Table 1 Clinical and radiological features and pulmonary function of patients with PI $Z$ phenotype

\begin{tabular}{|c|c|c|c|c|c|c|c|c|c|c|c|}
\hline \multirow{2}{*}{$\begin{array}{l}\text { Patient } \\
\text { no. }\end{array}$} & \multirow[t]{2}{*}{ Age } & \multirow[t]{2}{*}{ Sex } & \multirow{2}{*}{$\begin{array}{l}\text { Smoking } \\
\text { (pack/years) }\end{array}$} & \multirow{2}{*}{$\begin{array}{l}\text { Dyspnoea } \\
\text { grade }\end{array}$} & \multirow{2}{*}{$\begin{array}{l}A g e \\
C B\end{array}$} & \multicolumn{2}{|c|}{ Radiographic findings } & \multirow{2}{*}{$\begin{array}{l}\text { Bullae on } \\
\text { CT scan }\end{array}$} & \multirow[t]{2}{*}{ Hypoxaemia } & \multirow{2}{*}{$\begin{array}{l}F E V_{1} \\
\text { (\% predicted) }\end{array}$} & \multirow[t]{2}{*}{$T_{L C O}$} \\
\hline & & & & & & Hyperinflation & Bullae & & & & \\
\hline 1 & 35 & $M$ & 40 & 3 & 24 & +++ & + & + & Yes & 29 & $\downarrow$ \\
\hline 2 & 34 & M & 30 & 2 & 29 & + & - & + & No & 63 & $\downarrow$ \\
\hline 3 & 40 & M & 40 & 3 & 32 & +++ & + & + & Yes & 25 & $\downarrow$ \\
\hline 4 & 62 & $\mathrm{~F}$ & 0 & 3 & 37 & + & - & + & No & 73 & $\downarrow$ \\
\hline 5 & 40 & M & 8 & 3 & 36 & + & - & + & Yes & 38 & $\downarrow$ \\
\hline 6 & 66 & $\mathrm{~F}$ & 5 & 3 & 51 & + & - & + & Yes & 45 & $\downarrow$ \\
\hline
\end{tabular}

Age CB = age at which criteria of chronic bronchitis fulfilled; TLCO = carbon monoxide transfer factor.

in a pilot study of 102 newborn infants and 50 patients with COPD that no PI SZ individual was identified above this point.

In those individuals with suspect samples on immunodiffusion, a blood sample was taken and the serum phenotype was determined by isoelectrofocusing at $\mathrm{pH} 4.2-4.9$ on polyacrylamide gel $^{9}$ and the $\alpha_{1}$-antitrypsin serum level was determined by immunonephelometry (Beckman).

CLINICAL STUDY

Case histories were taken from all PI Z and PI SZ individuals who underwent physical examination and analysis of basic haematological and biochemical parameters including hepatic enzymes. PI $Z$ and PI SZ individuals from the population with COPD also underwent chest radiography, computed axial tomographic (CT) scanning, spirometric and pulmonary function tests.

DATA ANALYSIS

The comparison of prevalences was carried out using the Fisher exact test.

\section{Results}

There was a predominance of men $(79.8 \%)$, of smokers $(80.5 \%)$, and of those aged $40-60$ years $(57.4 \%)$ in the study population. Mean (SD) $\mathrm{FEV}_{1}$ was $1.14(0.32) 1$ (56 (17)\% predicted). $\mathrm{FEV}_{1} / \mathrm{FVC}$ was $54(14) \%$.

Of the 702 patients with COPD subjected to screening, $17(2.4 \%)$ were found to have an $\alpha_{1}$-antitrypsin/transferrin ratio of less than 0.65 . Phenotype determination showed that six of these were PI Z and one was PI SZ; the rest consisted of five PI MZ, three PI MS, and two PI MM. In the neonate population 58 (3.8/ 1000) suspect samples were detected of which eight were PI Z and 24 PI SZ; the remaining suspect samples consisted of one PI FS, three PI SS, five PI MZ, five PI MS, six PI MM, and six infants whose phenotype we were unable to determine. The number of PI $\mathrm{Z}$ individuals found in the group of patients with COPD was significantly higher $(\mathrm{p}<0.001)$ than that found in the neonates. The difference was not significant for PI SZ ( $\mathrm{p}=0.975)$.

The six COPD patients with PI Z presented with emphysema (table 1). Four subjects were aged $\leq 40$. All presented with radiological and CT findings compatible with emphysema. The PI SZ individual detected was a 60 year old man with clinical and spirometric criteria of
COPD five years previously. After his inclusion in the study population he was diagnosed as having extrinsic allergic alveolitis after exposure to avian antigens.

With respect to the neonate population, four of the eight PI $Z$ individuals detected presented with hepatomegaly; one child developed cirrhosis and portal hypertension, while the other three presented at one year with persistent hepatomegaly and raised hepatocellular enzymes. The remaining four PI $\mathrm{Z}$ individuals were asymptomatic with no biochemical changes. The 24 PI SZ neonates were also asymptomatic, and only one presented with slightly raised hepatocellular enzymes.

\section{Discussion}

Although the association between the $\mathrm{Pi} \mathrm{Z}$ phenotype and the development of pulmonary emphysema is well known, it is at present impossible to predict the natural history of a PI $Z$ individual. The number of PI $Z$ individuals with emphysema is well below the total number of these individuals in the population ${ }^{10}$ and, in most screening studies carried out on the general population, the PI $\mathrm{Z}$ individuals detected present with low levels of respiratory disease. ${ }^{11}$ This lack of knowledge is more marked when we consider PI SZ individuals. It is unclear whether this phenotype involves a risk of developing pulmonary emphysema. Further understanding on this matter is important as the number of PI SZ individuals in the population is 2-3 times greater than that of the PI Z phenotype.

In the controversy about the pulmonary involvement of this phenotype, an initial study described the association of PI SZ with pulmonary emphysema ${ }^{4}$ which resulted in PI SZ being considered a risk phenotype. However, the number of PI SZ individuals recorded with emphysema is clearly lower than the number of PI $Z$ individuals despite being a more frequent phenotype, and this suggests that the SZ phenotype does not involve any special risk for the development of respiratory disease. ${ }^{6}$

The number of studies on the prevalence of PI Z and PI SZ individuals in COPD populations is small. Only two studies have analysed populations of over 500 . Lieberman $^{7}$ found a prevalence of PI Z of $1.9 \%$ in 965 patients with COPD and reported no difference between these patients and normal individuals with regard to the number with the PI SZ phenotype. On the other hand, Bartman ${ }^{5}$ found five PI $Z$ and $18 \mathrm{PI} \mathrm{SZ}$ individuals in 526 patients with COPD compared with two PI $\mathrm{Z}$ and one PI 
SZ individuals in the control population. In our study we have found only one PI SZ individual among the patients with COPD, the same frequency as in our control group. However, because of the low prevalence of PI SZ in the population, larger studies may be necessary to exclude a small increase in COPD with PI SZ phenotype.

It was unknown before the beginning of our study whether PI SZ caused ill health so we chose neonates as a control population. In control groups of healthy adults the prevalence of PI Z and PI SZ may be underestimated because these individuals may develop initial symptoms in the second decade of life and are thus not adequately represented in control populations of adult groups.

Our study shows no involvement of the SZ phenotype in the risk for COPD. The frequency of COPD in PI $\mathrm{Z}$ individuals was far higher than that found in the neonate population ( $p$ $<0.001$ ), thus confirming the results of other studies with small populations of patients with COPD.

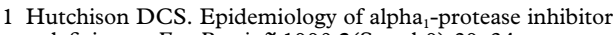
deficiency. Eur Respir f 1990;3(Suppl 9):29-34.

2 Gadek JE, Crystal RG. Alpha-1-antitrypsin deficiency. In Stanbury JB, Wyngaarden JB, Fredickson DS, Goldstein $\mathrm{JL}$, Brown MS, eds. The metabolic basis of inherited diseases. New York: McGraw-Hill,1982:1450-65.

3 Larsson C. Natural history and life expectancy in severe alpha $_{1}$-antitrypsin deficiency, Pi Z. Acta Med Scand 1978. 204:345-51.

4 Larsson C, Dirksen H, Sundstrom G, Eriksson S. Lung function studies in asymptomatic individuals with moderately (Pi SZ) and severely (PiZ) reduced levels of alpha1-antitrypsin. Scand $\mathcal{F}$ Respir Dis 1976;57:267-80.

5 Bartmann K, Fooke-Achterrath M, Koch G, Nagy I, Schültz $\mathrm{I}$, Weis $\mathrm{E}$, et al. Heterozygosity in the $\mathrm{Pi}$ system as a pathogenetic cofactor in chronic obstructive pulmonary disease (COPD). Eur f Respir Dis 1985;66:284-96.

6 Hutchison DCS, Tobin MJ, Cook PJL. Alpha-1-antitrypsin deficiency: clinical and physiological features in heterozygotes of Pi type SZ. A survey by the British Thoracic Association. Br f Dis Chest 1983;77:28-34.

7 Lieberman J, Winter B, Sastre A. Alpha antitrypsin Pi-types in 965 COPD patients. Chest 1986;89:370-3.

8 Laurell C-B. A screening test for $\alpha$-1-antitrypsin deficiency. Scand 7 Clin Lab Invest 1972;29:247-8.

9 Jeppsson J-O, Franzen B. Typing of genetic variants of $\alpha_{1}$. antitrypsin by electrofocusing. Clin Chem 1982;28:219-25.

10 Tobin MJ, Cook PJL, Hutchison DCS. Alpha-1-antitrypsin deficiency: the clinical and physiological features of pulmonary emphysema in subjects homozygous for Pi type Z. Br f Dis Chest 1983;77:14-27.

11 Morse JO, Lebowitz MD, Knudson RJ, Burrows B. Relation of protease inhibitor phenotypes to obstructive lung diseases in a community. $N$ Engl F Med 1977;296:1190-4. 